\title{
Effects of Custodiol-N, a novel organ preservation solution, on ischemia/reperfusion injury
}

\author{
Sivakkanan Loganathan, ${ }^{\mathrm{a}}$ Tamás Radovits, MD, PhD, ${ }^{\mathrm{a}, \mathrm{b}}$ Kristóf Hirschberg, MD, PhD, a,b \\ Sevil Korkmaz, PhD, ${ }^{a}$ Achim Koch, MD, ${ }^{a}$ Matthias Karck, MD, and Gábor Szabó, MD, PhD ${ }^{\mathrm{a}}$
}

Objective: Custodiol (histidine-tryptophan-ketoglutarate solution) is a leading organ preservation solution. On the basis of this solution, the novel Custodiol-N was developed. The present study investigated the effects of Custodiol-N in a rat model of heart transplantation.

\begin{abstract}
Methods: Heterotopic heart transplantation was performed in Lewis rats. Four groups were assigned: 2 Custodiol-N-treated groups and 2 Custodiol-treated control groups with a reperfusion time of 1 hour and 24 hours, respectively. Coronary blood flow, left ventricular pressure, its first derivative, left ventricular end-diastolic pressure, endothelium-dependent vasodilatation to bradykinin and endothelium-independent vasodilatation to sodium nitroprusside, and adenosine triphosphate content were measured. Terminal deoxynucleotidyl transferase-mediated dUTP-biotin nick end labeling staining was performed to detect apoptotic cardiomyocytes.
\end{abstract}

Results: After 1 hour, coronary blood flow $(3.99 \pm 0.24 \mathrm{~mL} / \mathrm{min} / \mathrm{g}$ vs $2.86 \pm 0.35 \mathrm{~mL} / \mathrm{min} / \mathrm{g} ; P<.05)$, left ventricular pressure $(117 \pm 18 \mathrm{~mm} \mathrm{Hg}$ vs $82 \pm 4 \mathrm{~mm} \mathrm{Hg} ; P<.05)$, and first derivative of left ventricular pressure $(3453 \pm 577 \mathrm{~mm} \mathrm{Hg} / \mathrm{s}$ vs $1740 \pm 116 \mathrm{~mm} \mathrm{Hg} / \mathrm{s} ; P<.05)$ were significantly higher in the Custodiol-N group compared with the corresponding control. The left ventricular systolic pressure-volume relationship was significantly steeper, indicating improved contractility. Vasodilatatory response to sodium nitroprusside did not show any major differences between the groups. Response to bradykinin resulted in a significantly higher increase in coronary blood flow in the Custodiol-N group $(92 \% \pm 4 \%$ vs $60 \% \pm 5 \% ; P<.05)$. Myocardial adenosine triphosphate content was significantly higher in the Custodiol-N group $(9.84 \pm 0.68 \mu \mathrm{mol} / \mathrm{g}$ vs $1.86 \pm 0.41$ $\mu \mathrm{mol} / \mathrm{g} ; P<.05)$. Terminal deoxynucleotidyl transferase-mediated dUTP-biotin nick end labeling staining showed a significantly reduced apoptosis level $(21.58 \% \pm 1.59 \%$ vs $27.23 \% \pm 1.54 \% ; P<.05)$ in the Custodiol-N group.

Conclusion: Custodiol-N improves myocardial and endothelial function during the critical phase of reperfusion after heart transplantation. (J Thorac Cardiovasc Surg 2010;139:1048-56)

To date, the major part of routine cardiac surgery is performed using extracorporal circulation with cardioplegic arrest. Independently of the technique of cardioplegia, temporary cardiac dysfunction can be observed frequently as a consequence of ischemia/reperfusion injury. In addition, coronary endothelial and peripheral vascular dysfunction may further complicate the postoperative course. Extracorporal circulation is also known to induce a systemic inflammatory reaction, with free radical release leading to secondary organ injury. The histidine-tryptophan-ketogluta-

From the Department of Cardiac Surgery, University of Heidelberg, ${ }^{a}$ Heidelberg, Germany; and Heart Center, Semmelweis University, ${ }^{\mathrm{b}}$ Budapest, Hungary.

This study was supported by Land Baden-Württemberg. There is no conflict of interest to declare.

Disclosures: G.S. reports grant support from Kohler Chemie, the manufacturer of the cardioplegic solutions.

Received for publication June 24, 2009; revisions received Sept 5, 2009; accepted for publication Sept 17, 2009; available ahead of print Nov 30, 2009.

Address for reprints: Sivakkanan Loganathan, Laboratory of Cardiac Surgery, Department of Cardiac Surgery, University of Heidelberg, Im Neuenheimer Feld 326 (2. OG), 69120 Heidelberg, Germany (E-mail: sivakkanan.loganathan@googlemail. com)

0022-5223/\$36.00

Copyright $\odot 2010$ by The American Association for Thoracic Surgery doi:10.1016/j.jtcvs.2009.09.034 rate solution (HTK) (Bretschneider's solution; Custodiol), developed by Bretschneider, ${ }^{1}$ has been established as a leading cardioprotective solution in the field of routine cardiac surgery and cardiac transplantation.

In the past 2 decades, it has been shown that not only ischemia but also reperfusion itself contributes to myocardial and endothelial injury. ${ }^{2}$ The pathophysiology of ischemia/ reperfusion injury is complex and involves the production and release of inflammatory mediators and oxygen free radicals, ${ }^{3}$ and activation of the coagulation cascade, fibrinolytic system, and complement chain. ${ }^{4,5}$ Another mechanism of cardiac damage after cardiac arrest in heart surgery is induced by hypothermia, which is mainly mediated by an iron-dependent formation of reactive oxygen species. ${ }^{6,7}$ The formation of highly reactive species is triggered by a cold-induced increase in the cellular chelatable iron pool.

A new, iron-independent component of cold-induced injury was recently described. This iron-independent, chloride-dependent cellular damage was considered a second-line injury, overshadowed by the iron-dependent component. ${ }^{8}$

A third mechanism of cardioplegia-induced tissue injury has its roots in one of the components used in Custodiol: histidine. Histidine has proven to be an excellent buffer and has 


\begin{tabular}{|c|c|}
\hline \multicolumn{2}{|c|}{ Abbreviations } \\
\hline ADP & $=$ adenosine diphosphate \\
\hline AMP & $=$ adenosine monophosphate \\
\hline ATP & $=$ adenosine triphosphate \\
\hline $\mathrm{dP} / \mathrm{dt}$ & $\begin{array}{l}=\text { first derivative of left ventricular } \\
\text { pressure }\end{array}$ \\
\hline $\mathrm{dP} / \mathrm{dt}_{\mathrm{ma}}$ & $\begin{aligned}= & \text { positive peak of the first derivative of } \\
& \text { left ventricular pressure }\end{aligned}$ \\
\hline $\mathrm{dP} / \mathrm{dt}_{\mathrm{mi}}$ & $\begin{aligned}= & \text { negative peak of the first derivative of } \\
& \text { left ventricular pressure }\end{aligned}$ \\
\hline HTK & $\begin{array}{l}=\text { histidine-tryptophan-ketoglutarate } \\
\text { solution }\end{array}$ \\
\hline LV & $=$ left ventricle \\
\hline$P_{\max }$ & $=$ left ventricular systolic pressure \\
\hline SNP & $=$ sodium nitroprusside \\
\hline TUNEI & $\begin{aligned}= & \text { terminal deoxynucleotidyl } \\
& \text { transferase-mediated dUTP-biotin } \\
& \text { nick end labeling }\end{aligned}$ \\
\hline
\end{tabular}

therefore been introduced into Custodiol. It was recently shown that this protective buffer itself can be the origin of cytotoxity. ${ }^{9}$ Like the chloride-dependent cellular damage, it is overshadowed by the iron-dependent formation of reactive oxygen species. ${ }^{10}$ But these mechanisms may become the main limiting factor for cell survival when the strong iron-dependent component is inhibited.

In a previous screening study, several solutions were tested in vitro to obtain the composition of Custodiol-N. ${ }^{10}$ Custodiol-N is a chloride- and histidine-poor cardioplegic solution based on Custodiol and supplemented by cytoprotective amino acids: LK614 and deferoxamine (Table 1). LK614 is a newly developed, lipophilic, membrane-permeable iron chelator quenching intracellular free iron. Deferoxamine is a well-known extracellular iron chelator. The present study compares the efficacy and safety of this newly developed cardioplegic solution with Custodiol in a model of heterotopic heart transplantation in the rat. The present study did not investigate the effect of each single component of the modified solution separately because their modes of action are well known. We aimed to assess the effects of the solution as a whole.

\section{MATERIALS AND METHODS}

\section{Animals and Experimental Groups}

Four groups were assigned: 2 Custodiol-N-treated groups and 2 Custodiol-treated control groups with reperfusion times of 1 hour and 24 hours, respectively. Six male Lewis rats weighing 250 to $300 \mathrm{~g}$ were used in each group. Target parameters were assessed after 1 hour of reperfusion and 24 hours of reperfusion. For the organ bath experiments, a Custodiol$\mathrm{N}$ group, a Custodiol group, and an additional nonischemic absolute control group were assigned. In each group, $\mathrm{n}=16$ aortic rings were assessed. All animals received humane care in compliance with the "Principles of Labo- ratory Animal Care," formulated by the National Society for Medical Research, and the "Guide for the Care and Use of Laboratory Animals," prepared by the Institute of Laboratory Animal Resources and published by the National Institutes of Health (Publication No. 86-23, revised 1996). The experiments were approved by the Ethical Committee of the Land Baden-Württemberg for Animal Experimentation.

\section{Heterotopic Heart Transplantation}

The donor rats were anesthetized with a single intraperitoneal injection of xylazine $(3 \mathrm{mg} / \mathrm{kg})$ and ketamine $(100 \mathrm{mg} / \mathrm{kg})$ and heparinized with $400 \mathrm{IU} / \mathrm{kg}$. The abdominal aorta was exposed, and a $1-\mathrm{cm}$ segment of the infrarenal aorta was occluded by small-vessel forceps. A polytetrafluorethylene catheter (Vygon GmbH \& Co. KG, 52017; Aachen, Germany) was advanced into the aorta. A bilateral thoracotomy was performed, and the heart was exposed. Cardiac arrest was induced by injection of $40 \mathrm{~mL}$ of Custodiol or Custodiol-N (Dr Franz Köhler Chemie GmbH; Alsbach-Hähnlein, Germany). After cardiac arrest, the superior and inferior caval veins and the pulmonary veins were tied en masse with a 4-0 single silk suture, and the heart was immediately placed into cold HTK solution $\left(4^{\circ} \mathrm{C}\right)$.

The recipient rats were anesthetized with a single intraperitoneal injection of xylazine $(3 \mathrm{mg} / \mathrm{kg})$ and ketamine $(100 \mathrm{mg} / \mathrm{kg})$ and heparinized with $400 \mathrm{IU} / \mathrm{kg}$. Two-centimeter segments of the infrarenal aorta and the caval vein were isolated and occluded by small-vessel forceps. The aorta and pulmonary artery of the donor heart were anastomosed end to side to the abdominal aorta and the vena cava, respectively, of the recipient rat. To minimize variability between experiments, the duration of the implantation was standardized at 60 minutes. After completion of the anastomoses, the vessels were released and the heart was then reperfused with blood in situ.

\section{Functional Measurement in the Graft}

After 60 minutes, a 3F latex balloon catheter (Edwards Lifesciences Corp, Irvine, CA) was introduced into the left ventricle (LV) via the apex and connected to a precision-calibrated syringe for administration and withdrawal of fluid to determine LV pressure, the first derivative $(\mathrm{dP} / \mathrm{dt})$, and end-diastolic pressure by a Millar micromanometer (Millar Instruments, Inc, Houston, TX) at different LV volumes. From these data, LV pressure-volume relationships were constructed using PVAN 3.6 software (Millar Instruments, Inc).

Coronary blood flow was measured by an ultrasonic flow meter mounted on the donor aorta. Endothelium-dependent vasorelaxation was evaluated by the peak coronary blood flow after a single bolus injection of bradykinin $\left(0.2 \mathrm{~mL}, 10^{-4} \mathrm{~mol} / \mathrm{L}\right)$ into the left of the graft. Endothelium-independent vasorelaxation was evaluated equally by a single bolus injection of sodium nitroprusside (SNP) $\left(0.2 \mathrm{~mL}, 10^{-2} \mathrm{~mol} / \mathrm{L}\right)$.

\section{In Vitro Organ Bath}

The organ bath experiments were performed as previously described. ${ }^{10}$ Briefly, the descending thoracic aorta was removed and cut transversely into rings $4 \mathrm{~mm}$ wide. Isolated aortic rings of the nonischemic group were investigated immediately. In the conservation groups, rings were placed in a test tube containing the different cardioplegic solutions $\left(4^{\circ} \mathrm{C}\right)$ The test tubes were closed, and the aortic rings were stored in the respective cardioplegic solution for 24 hours at $4^{\circ} \mathrm{C}$. The isolated aortic rings were mounted on stainless-steel hooks in individual organ baths containing Krebs-Henseleit solution. ${ }^{10}$

In Series I, the aortic rings were placed under a resting tension of $2 \mathrm{~g}$. Phenylephrine $\left(10^{-6} \mathrm{~mol} / \mathrm{L}\right)$ was used to pre-contract the rings, and relaxation responses were examined by adding cumulative concentrations of the endothelium-dependent dilator acetylcholine $\left(10^{-9}\right.$ to $\left.10^{-4} \mathrm{~mol} / \mathrm{L}\right)$ and endothelium-independent dilator SNP $\left(10^{-10}\right.$ to $\left.10^{-5} \mathrm{~mol} / \mathrm{L}\right)$. Because the major source of free radicals and oxidants produced during ischemia/reperfusion 
TABLE 1. Compounds of evaluated cardioplegic solutions

\begin{tabular}{lcc}
\hline & Custodiol mmol/L & Custodiol-N mmol/L \\
\hline $\mathrm{Na}^{+}$ & 15 & 16 \\
$\mathrm{~K}^{+}$ & 10 & 10 \\
$\mathrm{Mg}^{2+}$ & 4 & 8 \\
$\mathrm{Ca}^{2+}$ & 0.015 & 0.020 \\
$\mathrm{Cl}^{-}$ & 50 & 30 \\
$\mathrm{~L}-$-histidine & 198 & 124 \\
$\mathrm{~N}-\alpha$-acetyl-L-histidine & - & 57 \\
Tryptophan & 2 & 2 \\
A-ketoglutarate & 1 & 2 \\
Aspartate & - & 5 \\
Arginine & - & 3 \\
Alanine & - & 5 \\
Glycine & - & 10 \\
Mannitol & 30 & - \\
Sucrose & - & 33 \\
Deferoxamine & - & 0.025 \\
LK-614 & - & 0.0075 \\
\hline
\end{tabular}

Composition of the clinically used Custodiol and the newly developed Custodiol-N solution.

are activated leukocytes in vivo, which are absent in the present in vitro model, it was necessary to add an external oxidant source to the aortic rings to better simulate the clinical situation. Thus, in Series II, the aortic rings were investigated in a similar manner, with additional exposure to hypochlorite $(200 \mu \mathrm{mol} / \mathrm{L})$ for 30 minutes and rinsing before phenylephrine pre-contraction. Relaxation is expressed as the percentage of contraction induced by phenylephrine $\left(10^{-6} \mathrm{~mol} / \mathrm{L}\right)$.

\section{Histologic Analysis}

After excision, the hearts were incubated in $4 \%$ paraformaldehyde for histologic staining. The tissues were then fixed in an automatic tissue-fixing machine and embedded in paraffin. Apoptosis Detection Kit ApopTag Plus Peroxidase In Situ (Millipore Corporation, 290 Concord Road, Billerica, MA) was used for staining. Apoptosis detection is based on apoptosisinduced nuclear DNA fragmentation based on terminal deoxynucleotidyl transferase-mediated dUTP-biotin nick end labeling (TUNEL). Four watch fields were randomly selected in each section. Counting the cells was performed blinded. Positive and total cells were counted using Cell̂A software (Olympus Soft Imaging Solutions GmbH; Münster, Germany). Cells were considered apoptotic if more than $10 \%$ of the nucleus showed typical grey-brown color. Apoptosis index (=positive cells/total cells $\times \cdot 100 \%)^{11}$ was used as the indicator of apoptosis. To proof the correct procedure of the staining protocol, a positive control incubated with DNAase prior staining was provided.

\section{Determination of High-Energy Phosphates}

After excision, the hearts were immediately $(<1$ second) immersed in fluid nitrogen $\left(-196^{\circ} \mathrm{C}\right)$ and stored frozen at $-80^{\circ} \mathrm{C}$ until biochemical analysis. One gram of heart tissue was homogenized in $10 \mathrm{~mL}$ of $3.5 \% \mathrm{HCLO}_{4}$ and then centrifuged with $20,000 \mathrm{rpm}$ at $5^{\circ} \mathrm{C}$. Five milliliters of supernatant were neutralized with $1 \mathrm{~mL}$ of triethanolamine- $\mathrm{HCL} / \mathrm{K}_{2} \mathrm{CO}_{3}$ solution. Adenosine triphosphate (ATP) degradation was assessed with standard photometry using enzyme kinetic assay containing glycerinaldehyde-3-phosphate dehydrogenase, 3-phosphoglycerate-kinase, glycerin-3-phosphate dehydrogenase, and triosephosphate-isomerase. ATP, adenosine diphosphate (ADP), and adenosine monophosphate (AMP) contents were expressed as micromoles per gram of dry weight. Energy charge potential was calculated as $(\mathrm{ATP}+0.5 \mathrm{ADP}) /(\mathrm{ATP}+\mathrm{ADP}+\mathrm{AMP})$.

\section{Materials}

All materials used in our study were purchased from Sigma-Aldrich (St Louis, MO), unless otherwise specified. Custodiol and Custodiol-N (Table 1) were provided by Dr Franz Köhler Chemie GmbH.

\section{Statistical Analysis}

All values are expressed as mean \pm standard error of the mean. Individual means between the groups were compared by analysis of variance followed by an unpaired $t$ test with a Bonferroni correction for multiple comparisons.

\section{RESULTS}

\section{Functional Measurement in the Graft}

Left ventricular systolic pressure and peak positive $\mathrm{dP} / \mathrm{dt}$ were significantly $(P<.05)$ higher in the Custodiol-N group in comparison with the control group. Systolic cardiac function curves showed a significant leftward shift in this group (Figure 1, $A, B$ ). Peak negative $\mathrm{dP} / \mathrm{dt}$ was significantly higher $(P<.05)$, indicating a better myocardial relaxation (Figure 1, $D)$. After 24 hours of reperfusion, there were no significant differences between both groups in the previously mentioned hemodynamic parameters. Nevertheless, there was a slight tendency for higher values in left ventricular systolic pressure and $\mathrm{dP} / \mathrm{dt}_{\max }$ and $\mathrm{dP} / \mathrm{dt}_{\min }$ in the Custodiol-N group. Left ventricular end-diastolic pressure did not show any major differences among the 4 groups (Figure $1, C)$.

Coronary blood flow measurements showed a significant $(P>.05)$ increase after 1 hour of reperfusion compared with the corresponding control. After 24 hours of reperfusion, coronary blood flow in the Custodiol-N group remained high but did not show any significant differences. Assessment of endothelium-dependent vasorelaxation as an effect of bradykinin application showed a significantly increased vasodilatory response. Meanwhile, endothelium-independent vasorelaxation after application of SNP did not show significant differences (Figure 2).

\section{In Vitro Organ Bath}

After 24-hour hypothermic storage in Custodiol, a marked impairment of endothelial function was demonstrated the in vitro organ bath experiments. The endothelial dysfunction was indicated by the reduced maximal relaxation of isolated aortic rings to acetylcholine and the rightward shift of the dose-response curve compared with the nonischemic absolute control group.

In the case of long-term storage in the new cardioplegic solution, enhanced endothelium-dependent vasorelaxation to acetylcholine was detected when compared with the Custodiol group (Figure 3, $A, C$ ). In addition, $\mathrm{pD}_{2}=-\log \mathrm{EC}_{50}$ (M) $(\mathrm{EC} 50=$ half maximal response $)$ to acetylcholine showed significant improvements in the Custodiol-N group in both series without $\mathrm{NaOCl}$ incubation $(7.33 \pm 0.07 \#$ vs $7.03 \pm 0.09^{*} \#$ vs $6.69 \pm 0.07 *$; control vs Custodiol-N vs Custodiol; ${ }^{*} P<.05$ vs control; ${ }^{\#} P<.05$ vs Custodiol) and 

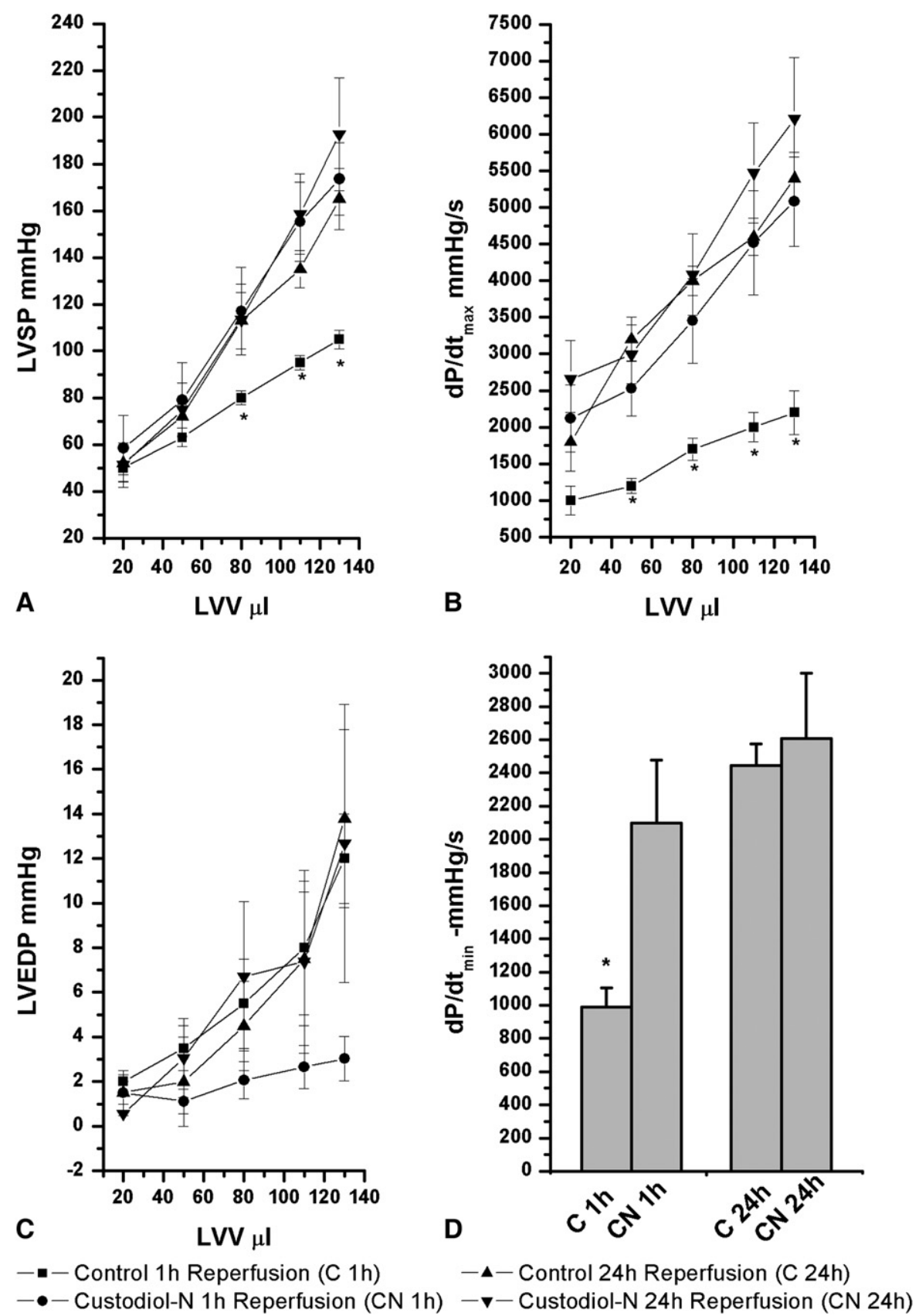

FIGURE 1. Hemodynamic analysis. A, Left ventricular peak systolic pressure - left ventricular volume. B, Maximum pressure development (dP/dtmax) left ventricular volume. C, Left ventricular end-diastolic pressure - left ventricular volume. D, Minimum pressure development (dP/dtmin) at an intraventricular volume of $80 \mu \mathrm{L}$ after 1 hour of reperfusion. All values are given as mean \pm standard error of the mean (SEM). $* P<.05$ vs other groups. LVSP, Left ventricular peak systolic pressure; $L V V$, left ventricular volume; $L V E D P$, left ventricular end-diastolic pressure.

with $\mathrm{NaOCl}$ incubation $\left(7.33 \pm 0.07\right.$ vs $6.88 \pm 0.16^{*}$ vs 6.75 $\pm 0.09 *$; control vs Custodiol-N vs Custodiol; $* P<.05$ vs control). As expected, endothelial function showed a stronger impairment after $\mathrm{NaOCl}$ incubation. In regard to the endothelium-independent vascular smooth muscle function of the aortic rings, which was indicated by endotheliumindependent vasorelaxation with SNP (Figure 3, $B, D$ ), there were no significant differences among the groups. Nevertheless, $\mathrm{pD}_{2}$ to $\mathrm{SNP}$ showed enhancements in the Custodiol-N group $(8.27 \pm 0.04 *$ vs $8.55 \pm 0.07$ vs $8.17 \pm 0.04 *$; control vs Custodiol-N vs Custodiol; $* P<.05$ vs Custodiol-N). After $\mathrm{NaOCl}$ incubation, no significant differences remained among the groups $(8.27 \pm 0.04$ vs $8.43 \pm 0.09$ vs 8.25 \pm 0.06; control vs Custodiol-N vs Custodiol). 

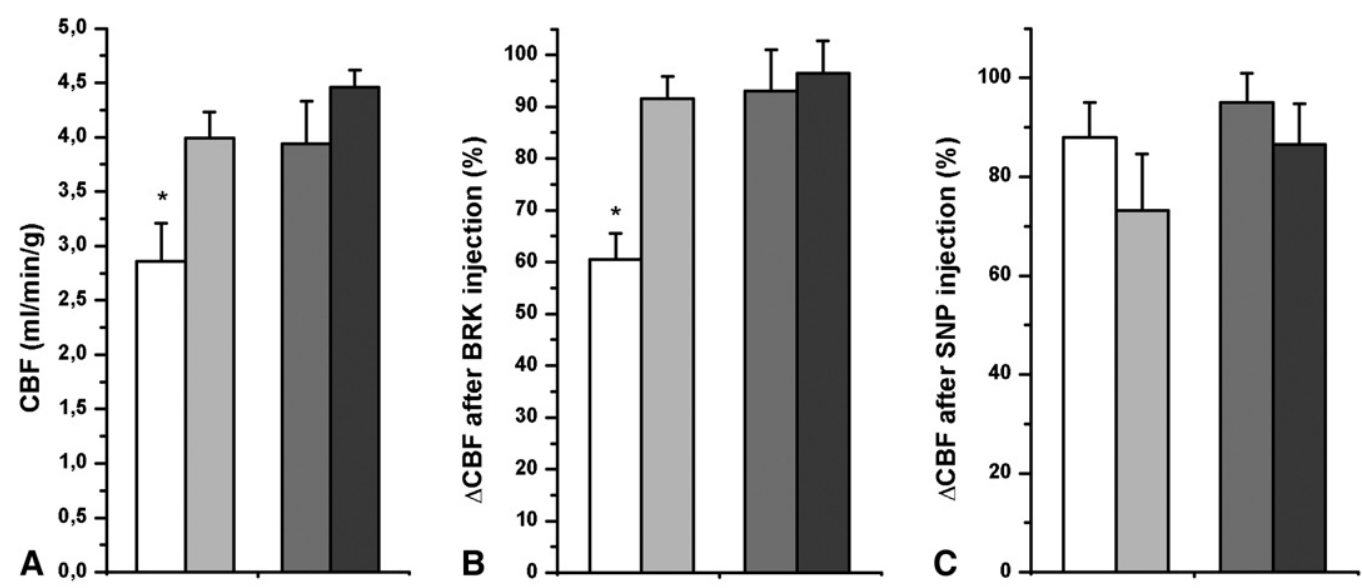

Custodiol 1h Reperfusion Custodiol-N 1h Reperfusion

FIGURE 2. Coronary blood flow. Baseline coronary blood flow (A) and vasodilator response after application of the endothelium-dependent vasodilator bradykinin $(0.1 \mathrm{nmol}, 0.2 \mathrm{~mL}, \mathrm{~B})$ and the endothelium-independent vasodilator SNP $(10 \mathrm{nmol}, 0.2 \mathrm{~mL}, \mathrm{C})$. All values are given as mean $\pm \mathrm{SEM}$. $* P<$ .05 vs corresponding control. $C B F$, Coronary blood flow; $B R K$, bradykinin; $S N P$, sodium nitroprusside.

\section{Histologic Analysis}

Figure 4 shows representative TUNEL staining in the control and Custodiol-N groups after 1 hour and 24 hours of reperfusion. Few apoptotic cells were found in the Custodiol-N group. In contrast, control group samples showed many TUNEL-positive nuclei. These results were confirmed by semiquantitative analysis of the apoptosis index. Figure 4, $E$ shows that the apoptosis index in control group rats was significantly higher than in the Custodiol-N group after 1 hour of reperfusion. After 24 hours of reperfusion, the apoptosis indices of both groups increased. Nevertheless, there were still significantly fewer apoptotic cells in the Custodiol-N group than in the control group.

\section{Biochemical Analysis}

Myocardial high-energy phosphate contents, especially ATP content, were preserved with Custodiol-N during heart transplantation (Table 2). ADP levels did not significantly differ among the different groups. Nevertheless, there was a strong tendency for higher ADP levels in the Custodiol$\mathrm{N}$ group. Concomitantly, AMP levels were significantly lower in this group compared with the corresponding control group. Energy charge potential as an indicator of the myocardial energy level showed a significant improvement with the novel Custodiol-N solution.

\section{DISCUSSION}

Presently, the major part of cardiac surgery relies on cardiac arrest induced by different cardioplegic solutions. Bretschneider's solution (Custodiol) was developed for organ conservation, but it is also widely used as a cardioplegic solution in routine cardiac surgery. Despite the capability of Bretschneider's solution (Custodiol) to effectively reduce energy requirements, ${ }^{12}$ it has been shown that damages occurring during reperfusion are left unaddressed by this cardioplegic solution and other solutions. Several pathways of oxidative stress have been implicated in the pathophysiology of severe damage after reperfusion. The fundamental pathway of cardiac damage during this phase is the production of superoxide anion, hydrogen peroxide, and possibly singlet oxygen. ${ }^{13}$ In support of the previously mentioned agents, reactive oxygen species formed in an iron-dependent manner have been discovered to play a crucial role in coldinduced injury. ${ }^{14}$ Recent studies ${ }^{10}$ clearly confirm the inability of the HTK solution (Custodiol) to reduce reperfusion injury after long-term cold ischemic storage. Custodiol-N addresses this issue using LK-614 and the well-known deferoxamine as iron chelators quenching free iron (Table 1).

LK614 [1-(N-hydroxy-N-methylcarbamide)-3,4-dimethoxybenzol] is a newly developed iron chelator. The aromatic ring of LK614 concedes enough lipophilicity to allow membrane permeability. ${ }^{15}$ Because of the combination of LK614 and deferoxamine, Custodiol-N reduces the load of free intracellular and extracellular $\mathrm{Fe}^{3+}$, leading to a strong reduction of hydroxyl radicals and iron-dependent reactive oxygen species, again leading to improved myocardial protection because of the reduction of oxidative stress. ${ }^{10}$

The amino acid histidine is an excellent buffer and therefore used as a "physiologic" buffer in the HTK solution. However, it was recently reported that histidine contributes decisively to preservation solution toxicity. The mechanism behind this toxicity has not been completely solved, but it has been proved that this mechanism is linked to an increased production of iron-dependent reactive oxygen species, most likely by histidine entering the cells and 

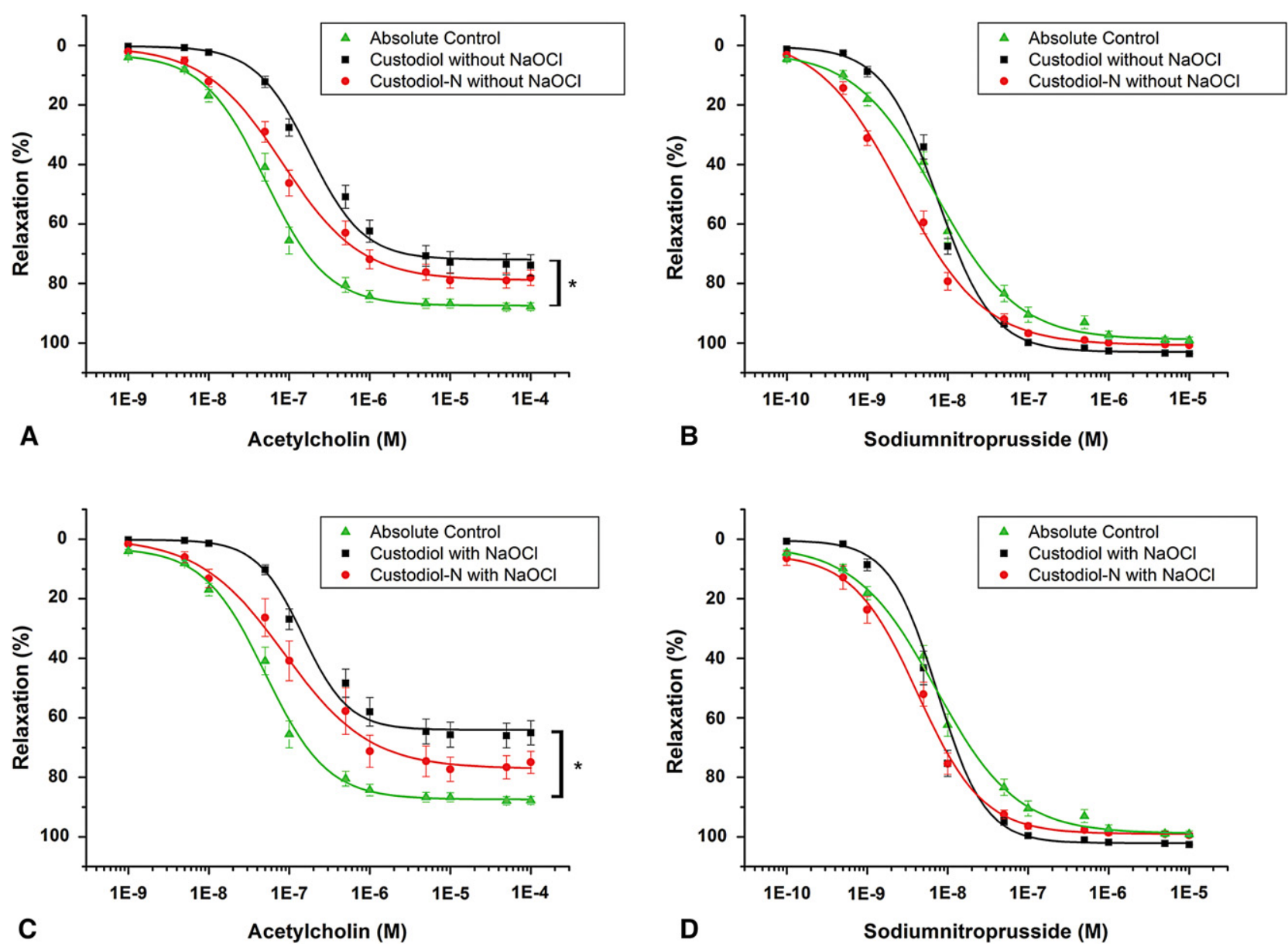

FIGURE 3. Vascular function after 24-hour cold storage. Concentration-response curves of acetylcholine. A, Acetylcholine-induced endotheliumdependent vasorelaxation in aortic rings after 24-hour cold ischemic storage. B, SNP-induced endothelium-independent vasorelaxation in aortic rings after 24-hour cold ischemic storage. C, Acetylcholine-induced endothelium-dependent vasorelaxation in aortic rings after 24-hour cold ischemic storage and additional $\mathrm{NaOCl}$ preincubation. D, SNP-induced endothelium-independent vasorelaxation in aortic rings after 24-hour cold ischemic storage and additional $\mathrm{NaOCl}$ preincubation. Each point on the curves and each column represent the mean $\pm \mathrm{SEM}$ of 16 experiments in thoracic aortic rings of the different groups. $* P<.05$ vs absolute control.

forming redox-active complexes with cellular chelatable iron and thereby forming reactive oxygen species. ${ }^{9}$ Apart from its toxicity, histidine has proven to be an excellent buffer. $\quad N$ - $\alpha$-acetyl-L-histidine, a naturally occurring ${ }^{16}$ $\mathrm{N}$-substituted derivate of histidine, is an attractive buffer to replace histidine. It shares the benefits of histidine and has almost no toxicity. ${ }^{9}$ The reason for this is most likely the decreased cellular uptake of $N$ - $\alpha$-acetyl-L-histidine and its altered affinity for iron. This issue was addressed by partially replacing histidine by $\mathrm{N}-\alpha$-acetyl-L-histidine in Custodiol- $\mathrm{N}$ (Table 1).

Iron-independent cellular damage dependent on extracellular chloride concentrations was recently described. ${ }^{8}$ However, the underlying molecular mechanisms remain unclear. Nevertheless, it has been hypothesized that the $\mathrm{Cl}^{-} / \mathrm{HCO}_{3}{ }^{-}$ exchanger most likely is involved in the mechanism of apoptosis. The $\mathrm{Cl}^{-}$efflux from cells or $\mathrm{HCO}_{3}{ }^{-}$influx to cells (consequently increasing the $\mathrm{pH}$ level) may play an important role in signal transduction leading to events such as activation of caspase, which again is an important inductor of apoptosis. ${ }^{17}$ This mechanism was taken into consideration, and the $\mathrm{Cl}^{-}$-concentration in Custodiol-N was reduced and partially replaced by aspartate (Table 1). Thus, increasing $\mathrm{pH}$ levels and the activation of caspase (in a final step) were reduced, also leading to a reduction of apoptosis in myocardial tissue and conservation of myocardial function after cold storage.

Another point that has been taken into account in the development of Custodiol- $\mathrm{N}$ is the protective effects of some amino acids, such as glycine, alanine, and arginine. The mechanism of action of glycine in cytoprotection of cells exposed to hypoxia or cold ischemia has been suggested to be based on the stabilizing effect of the plasma membrane. Glycine suppresses the release of intracellular 

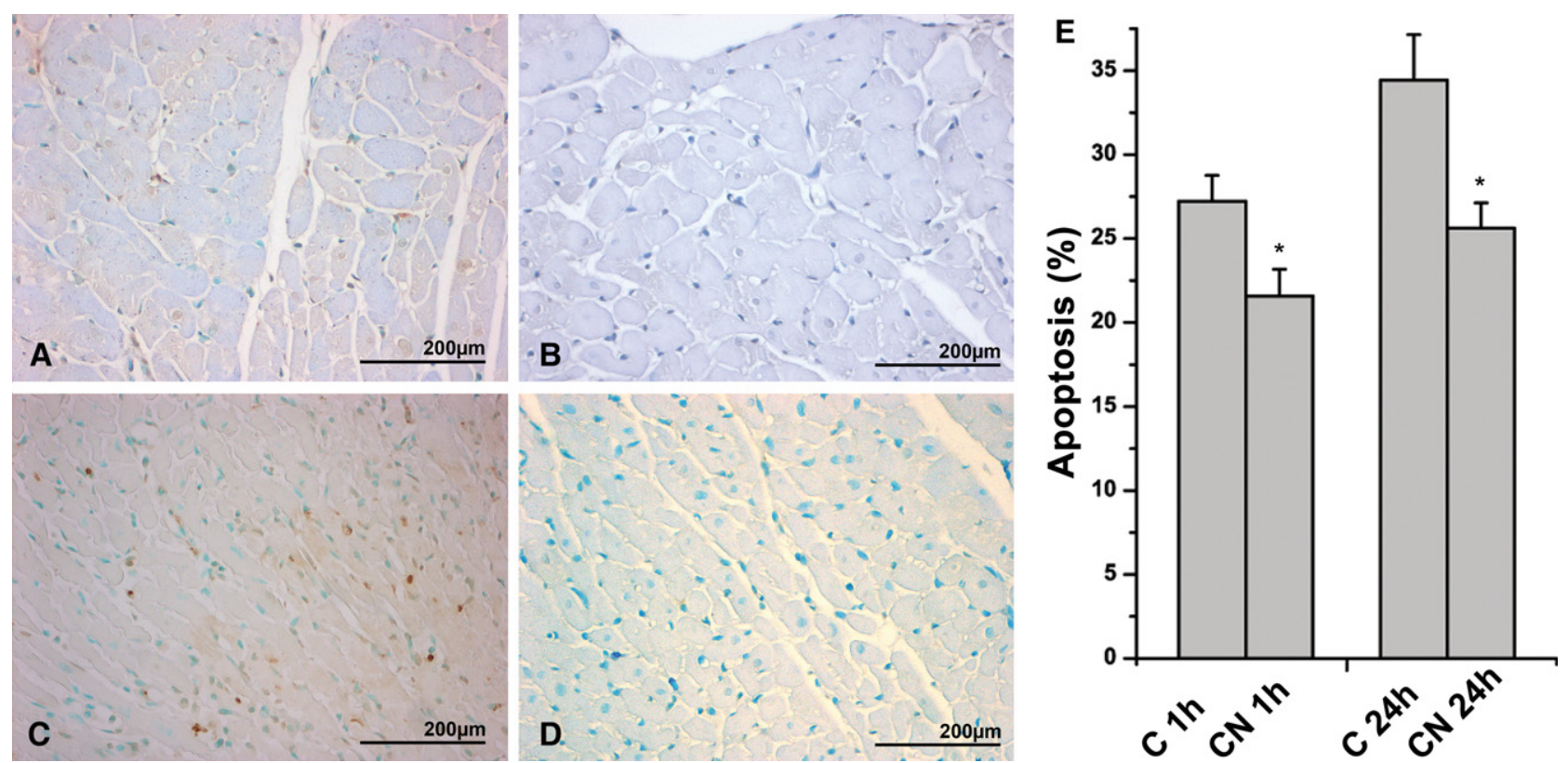

FIGURE 4. Histologic analysis. TUNEL staining after 1 hour and 24 hours of reperfusion; Control group also shows high apoptosis rate (grey-brown-colored nuclei) after 1 hour (A) and 24 hours (C). Custodiol-N group rarely shows any apoptotic cells after 1 hour (B) and 24 hours (D); apoptosis index (E). Control after 1-hour reperfusion versus Custodiol-N after 1-hour reperfusion and control after 24-hour reperfusion versus Custodiol-N after 24-hour reperfusion. Values are given as mean \pm SEM. $* P<.05$ vs corresponding control; $200 \times$ magnification. $C N$, Control.

enzymes of damaged cells. It has been suggested that this membrane-stabilizing effect of glycine is mediated by specific membrane-bound receptors. ${ }^{18}$ However, the protective pathway still remains unclear and requires further investigation to be understood sufficiently. Although the mechanism of cytoprotection is still unclear for glycine, this amino acid has proven its beneficial effects on ischemia/reperfusion injury and has therefore been introduced into Custodiol-N.

Alanine was supplemented in Custodiol-N because this amino acid is known to inhibit the hypoxia-associated sodium influx into the myocytes. During ischemia, the formation of unspecific membrane pores leads to intracellular sodium accumulation followed by chloride influx, cell swelling, and consequent cellular damage. ${ }^{19}$ By adding this amino acid, this negative course of cell swelling and apoptosis was decelerated.

Arginine is the precursor of nitric oxide. The beneficial effects of nitric oxide, such as increasing the postischemic blood flow, decreasing leukocyte adhesion, and expressing cell adhesion molecules, have been well explored. The administration of arginine improves the production of nitric oxide, especially in conditions with endothelial injury with subsequent diminished basal endothelial nitric oxide production (eg, in ischemia/reperfusion) ${ }^{20}$

In addition to these specific protective effects of the previously mentioned amino acids, all amino acids have buffering capabilities, which are important in organ preser- vation solutions. These additional buffering capabilities advance the specific protective effects of each amino acid. ${ }^{21}$

The focus of this study was the protection of the myocardial tissue against the hypothermic damage during cardioplegic arrest associated with Bretschneider's solution (Custodiol). Therefore, this solution was modified in 4 crucial ways: 1) addition of deferoxamine and LK614 as iron chelators to reduce the iron-dependent production of reactive oxygen species, 2) substitution of histidine by $\mathrm{N}$ - $\alpha$-acetyl-1-histidine to inhibit the histidine-associated forming of redox active complexes, 3 ) reduction of the chloride concentration to diminish $\mathrm{Cl}^{-}$-activated induction of apoptosis, and 4) addition of cytoprotective amino acids. ${ }^{18-21}$

In support of our results, these mechanisms show improved myocardial protection after cardioplegic arrest and

TABLE 2. Biochemical analysis

\begin{tabular}{lcccc}
\hline & $\begin{array}{c}\text { Control } \\
\text { 1-h } \\
\text { reperfusion }\end{array}$ & $\begin{array}{c}\text { Custodiol-N } \\
\text { 1-h reperfusion }\end{array}$ & $\begin{array}{c}\text { Control } \\
\text { 24-h reperfusion }\end{array}$ & $\begin{array}{c}\text { Custodiol-N } \\
\text { 24-h reperfusion }\end{array}$ \\
\hline ATP & $1.86 \pm 0.41$ & $9.84 \pm 0.68^{*}$ & $2.65 \pm 0.49$ & $8.05 \pm 1.62^{*}$ \\
ADP & $2.05 \pm 0.42$ & $3.07 \pm 0.30$ & $3.35 \pm 0.55$ & $3.28 \pm 0.41$ \\
AMP & $2.07 \pm 0.22$ & $0.91 \pm 0.13^{*}$ & $0.49 \pm 0.14$ & $0.83 \pm 0.14^{*}$ \\
ECP & $0.49 \pm 0.04$ & $0.82 \pm 0.01^{*}$ & $0.53 \pm 0.05$ & $0.79 \pm 0.01^{*}$ \\
\hline
\end{tabular}

$A T P$, Adenosine triphosphate; $A D P$, adenosine diphosphate; $A M P$, adenosine monophosphate; $E C P$, energy charge potential. All values are given as mean \pm SEM. ${ }^{*} P<.05$ vs corresponding control. 
recovery of myocardial function; the more rapid recovery of the hemodynamic parameters in comparison with Bretschneider's solution (Custodiol) especially shows the effectiveness of these modifications (Figure 1). After 24 hours, we were not able to record any further improvement using Custodiol-N. These data lead to the hypothesis that the Custodiol-N group reaches almost full functional recovery after 1 hour of reperfusion. This hypothesis is also supported by the data of one of our previous studies in which we performed a nonischemic heart transplantation using a slightly modified operation technique. ${ }^{22}$ This group showed similar hemodynamic results as the Custodiol-N group after 1 hour of reperfusion.

However, our biochemical data (Table 2) also indicate an improved myocardial energy state during early and late reperfusion. Nevertheless, the advancements of Custodiol$\mathrm{N}$ seem to be more apparent during early reperfusion. The slight reduction in high-energy phosphate content after 24 hours in comparison with 1 hour in the Custodiol-N group (Table 2) may be explained by myocardial unloading and a consequent adaption of the myocardium to these changed conditions.

This idea of myocardial protection during early and late reperfusion is also supported by the results of our histologic analysis (Figure 4, $A-D$ ). TUNEL staining is sensitive to double-strand breaks in the DNA of the nucleus; subsequently, this method is sensitive to the damage caused by reactive oxygen species inducing such damage and thereby also inducing apoptosis in a final step. The histologic analysis revealed that Custodiol-N powerfully reduced this type of damage and apoptosis (Figure 4,E).

In addition to our previous results, coronary blood flow measurement and in vitro organ bath experiments show that not only myocardial tissue but also endothelium benefited from these additional protection mechanisms (Figure 2). This is in accordance with a recent report of our research group showing the beneficial effects of iron chelators in cold storage-induced damage on endothelial dysfunction. ${ }^{10}$ The preservation of endothelial function and thereby an increased coronary blood flow may be partly responsible for the fast myocardial recovery and the improved myocardial energy state in our study.

\section{CONCLUSIONS}

These modifications of Custodiol can effectively reduce myocardial and endothelial damage after ischemia/reperfusion; however, functional improvements mainly occur during the early reperfusion phase. The phase of late reperfusion did not show any major differences in the functional parameters. The reduction of cardiac impairment, especially during the critical phase of early reperfusion, is known to improve the postoperative outcome. ${ }^{23}$ The mechanisms addressed by our modifications have been reported and described by other research groups, as well by our own group. ${ }^{6,8-10,14,17,24}$ Nevertheless, these findings have not been implemented in a cardioplegic solution. Custodiol-N is a novel cardioplegic solution showing how effectively a "cocktail" of many minor modifications, which have been analyzed extensively one by one, ${ }^{6,8-10,14,17,24}$ can protect myocardial tissue during ischemia/reperfusion. Our data indicate that Custodiol-N may have beneficial effects in patients undergoing surgical interventions on the heart with cardioplegic arrest.

\section{References}

1. Bretschneider HJ. Myocardial protection. Thorac Cardiovasc Surg. 1980;28: 295-302.

2. Farb A, Kolodgie FD, Jenkins M, Virmani R. Myocardial infarct extension during reperfusion after coronary artery occlusion: pathologic evidence. J Am Coll Cardiol. 1993;21:1245-53.

3. Sellke FW, Shafique T, Ely DL, Weintraub RM. Coronary endothelial injury after cardiopulmonary bypass and ischemic cardioplegia is mediated by oxygenderived free radicals. Circulation. 1993;88(5 Pt 2):II395-400.

4. Yamaguchi Y, Okabe K, Liang J, Ohshiro H, Ishihara K, Uchino S, et al. Thrombin and factor Xa enhance neutrophil chemoattractant production after ischemia/ reperfusion in the rat liver. J Surg Res. 2000;92:96-102.

5. Golino P, Ragni M, Cirillo P, Avvedimento VE, Feliciello A, Esposito N, et al Effects of tissue factor induced by oxygen free radicals on coronary flow during reperfusion. Nat Med. 1996;2:35-40.

6. Rauen U, Petrat F, Li T, De Groot H. Hypothermia injury/cold-induced apoptosis-evidence of an increase in chelatable iron causing oxidative injury in spite of low O2-/H2O2 formation. FASEB J. 2000;14:1953-64.

7. Salahudeen AK, Huang H, Patel P, Jenkins JK. Mechanism and prevention of cold storage-induced human renal tubular cell injury. Transplantation. 2000;70: 1424-31.

8. Rauen U, Kerkweg U, de Groot H. Iron-dependent vs iron-independent cold-induced injury to cultured rat hepatocytes: a comparative study in physiological media and organ preservation solutions. Cryobiology. 2007;54:77-86.

9. Rauen U, Klempt S, de Groot H. Histidine-induced injury to cultured liver cells, effects of histidine derivatives and of iron chelators. Cell Mol Life Sci. 2007;64: 192-205.

10. Radovits T, Lin LN, Zotkina J, Koch A, Rauen U, Kohler G, et al. Endothelia dysfunction after long-term cold storage in HTK organ preservation solutions: effects of iron chelators and N-alpha-acetyl-L-histidine. J Heart Lung Transplant. 2008;27:208-16.

11. Qian HY, Yang YJ, Huang J, Gao RL, Dou KF, Yang GS, et al. Effects of Tong xinluo-facilitated cellular cardiomyoplasty with autologous bone marrow-mesenchymal stem cells on postinfarct swine hearts. Chin Med J (Engl). 2007;120: 1416-25.

12. Hachida M, Ookado A, Nonoyama M, Koyanagi H. Effect of HTK solution for myocardial preservation. J Cardiovasc Surg (Torino). 1996;37:269-74.

13. Kukreja RC, Hess ML. The oxygen free radical system: from equations through membrane-protein interactions to cardiovascular injury and protection. Cardiovasc Res. 1992;26:641-55.

14. Rauen U, de Groot H. New insights into the cellular and molecular mechanisms of cold storage injury. J Investig Med. 2004;52:299-309.

15. Wille T, de Groot H, Rauen U. Improvement of the cold storage of blood vessels with a vascular preservation solution. Study in porcine aortic segments. $J$ Vasc Surg. 2008;47:422-31.

16. Drescher MJ, Drescher DG. N-acetylhistidine, glutamate, and beta-alanine are concentrated in a receptor cell layer of the trout inner ear. J Neurochem. 1991; 56:658-64.

17. Fujita H, Ishizaki Y, Yanagisawa A, Morita I, Murota SI, Ishikawa K. Possible involvement of a chloride-bicarbonate exchanger in apoptosis of endothelial cells and cardiomyocytes. Cell Biol Int. 1999;23:241-9.

18. Weinberg JM, Venkatachalam MA, Garzo-Quintero R, Roeser NF, Davis JA Structural requirements for protection by small amino acids against hypoxic injury in kidney proximal tubules. FASEB J. 1990;4:3347-54.

19. Bahde R, Palmes D, Gemsa O, Minin E, Stratmann U, de Groot H, et al. Attenuated cold storage injury of rat livers using a modified HTK solution. J Surg Res. 2008; 146:49-56. 
20. Soos P, Andrasi T, Buhmann V, Kohl B, Vahl C, Hagl S, et al. Myocardial protection after systemic application of L-arginine during reperfusion. J Cardiovasc Pharmacol. 2004;43:782-8.

21. Churchill TA, Green CJ, Fuller BJ. Protective properties of amino acids in liver preservation: effects of glycine and a combination of amino acids on anaerobic metabolism and energetics. J Hepatol. 1995;23:720-6.

22. Loganathan S, Radovits T, Hirschberg K, Korkmaz S, Barnucz E, Karck M, et al. Effects of selective phosphodiesterase-5-inhibition on myocardial contractility and reperfusion injury after heart transplantation. Transplantation. 2008;86: 1414-8.

23. Szabo G, Bahrle S, Stumpf N, Sonnenberg K, Szabo EE, Pacher P, et al. Poly(ADP-Ribose) polymerase inhibition reduces reperfusion injury after heart transplantation. Circ Res. 2002;90:100-6.

24. Okada Y, Shimizu T, Maeno E, Tanabe S, Wang X, Takahashi N. Volumesensitive chloride channels involved in apoptotic volume decrease and cell death. J Membr Biol. 2006;209:21-9. 